\title{
Corela
}

Cognition, représentation, langage

HS-30 | 2020

Phonétique, littérature et enseignement du FLE :

théories et recherches

\section{Propositions pour exploiter la poésie en classe de FLE : (re)construire le poème des sons et sa musicalité}

René Corona

\section{(2) OpenEdition}

Journals

Édition électronique

URL : http://journals.openedition.org/corela/10549

DOI : 10.4000/corela. 10549

ISSN : 1638-573X

Éditeur

Cercle linguistique du Centre et de l'Ouest - CerLICO

Référence électronique

René Corona, «Propositions pour exploiter la poésie en classe de FLE : (re)construire le poème des sons et sa musicalité », Corela [En ligne], HS-30 | 2020, mis en ligne le 02 mai 2020, consulté le 23 mai 2020. URL : http://journals.openedition.org/corela/10549 ; DOI : https://doi.org/10.4000/corela.10549

Ce document a été généré automatiquement le 23 mai 2020.

Corela - cognition, représentation, langage est mis à disposition selon les termes de la licence Creative Commons Attribution - Pas d'Utilisation Commerciale - Partage dans les Mêmes Conditions 4.0 International. 


\title{
Propositions pour exploiter la poésie en classe de FLE : (re)construire le poème des sons et sa musicalité
}

\author{
René Corona
}

\section{Hésitation des signifiants sur des vagues de signifiés}

1 Si le poème est, d'après Paul Valéry (2001:265), " cette hésitation prolongée entre le son et le sens ", - puisque le «euh" hésitant nous dit Bertrand Lauret (2007:45) «illustre une volonté de pratiquer/partager un geste vocal propre au français »-, et si, pour Guimbretière (1994 : 4), "l'oral, c'est cette partie de la langue qui devient parole », quoi de mieux que d'essayer de dégager quelques clefs de lecture afin de pratiquer le poème en classe de phonétique, ou moins prétentieusement, d'utiliser le poème pour jouer avec les sons (et les sens / l'essence du poème) dans un laboratoire de langue

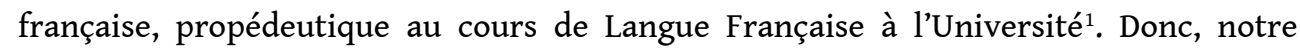
point de départ se voudra essentiellement du côté de la poétique, avec en regard la phonétique. Les deux iront ensemble comme le flux et le reflux de la marée, s'épouseront d'une certaine façon, dans le but de faire progresser et d'effacer, par le jeu et la poésie, les défauts accumulés au cours des années d'un secondaire italien souvent stigmatisé par de mauvais enseignements ou un manque de préparation et de temps, au cours d'années scolaires turbulentes: absence de professeurs, grèves, etc. Lorsque le nombre d'années d'études du français est de huit années (trois de scuola media et cinq de superiori), l'étudiant a tendance à considérer, du moins les trois premières années du collège comme insignifiantes et non concluantes. Les propositions suivantes visent des apprenants du Français Langue Étrangère de niveau intermédiaire à avancé, voire très avancé (de $\mathrm{B} 2$ à $\mathrm{C} 2$ selon le Cadre européen commun de référence pour les 
langues). Quel que soit le niveau des apprenants, l'enseignant doit remédier à des distorsions phonétiques et prosodiques avant que celles-ci ne se fossilisent.

Ne pourrions-nous donc pas conjuguer ce que Genette ${ }^{2}$, inspiré par Bachelard, nommait la « poétique du langage » avec la phonétique des mots dans une classe de $\mathrm{FLE}^{3}$ ? Pour notre cours de FLE, il y a deux éléments qui pourront nous être utiles : le premier est ce que Genette, après Mallarmé et Jakobson, dit à propos du jour et de la nuit, l'autre est ce qu'écrit Élisabeth Guimbretière (ibid. : 41) : « le locuteur se sert souvent de la courbe mélodique pour renforcer le sémantisme d'un énoncé mais aussi pour le contredire ». Musicalité et sens.

Commençons par le deuxième élément : l'oralité du poème passe aussi à travers cette mélodie que le récitant peut utiliser à son gré pour mieux connoter le vers, voire le sortilège du vers, pour reprendre le titre d'un vieil essai sur la poésie qui est encore actuel $^{4}$. Sonorités, rythmes et lectures à haute voix. Citons le Narrateur enfant de Proust qui rêve sur le nom des villes ${ }^{5}$ ou Jean-Paul Sartre qui explique la ville-fleur Florence ${ }^{6}$, ainsi que les vers d'Alexander Pope (cité par Jakobson), accompagnés par les vers qui le précèdent et ceux qui suivent: "Tis not enough no harshness gives offence / The sound must seem an echo to the sense. / Soft is the strain when Zephyr gently blows, / But when loud surges lash the sounding shore ${ }^{7}$ ». Puisqu'au centre de notre cours poético-phonétique se trouve, nécessairement, la traduction - sur laquelle nous ne nous attarderons pas ici -, il est à remarquer que les vers de Pope traduits en note sont deux traductions qui représentent assurément les deux courants : sourciers et ciblistes, les premiers prônant une traduction liée plus à la lettre, les seconds étant plus proches de l'esprit du texte ${ }^{8}$. Il faut donc que les apprenants de FLE découvrent que la bonne prononciation d'une langue peut aussi passer par la lecture d'un poème et, pour peu que nos apprenants aient plus de passion pour la chanson que pour la poésie, commencer par celle-ci pour arriver à celui-là.

Mais, si nous revenons à notre premier élément, notre point de départ sera le suivant : la langue cache des mystères qui nous échappent et nous prendrons comme exemple la phrase de Mallarmé à propos du jour et de la nuit. Lire, tout d'abord, la prose mallarméenne peut dérouter l'apprenant, mais il faut qu'il apprenne-ou qu'il réapprenne, s'il n'a pas oublié les comptines de la maternelle - que les mots ne sont pas simplement du matériau communicatif et que, derrière les mots, autre chose peut être celé :

mais sur l'heure, tourné à de l'esthétique, mon sens regrette que le discours défaille à exprimer les objets par des touches y répondant en coloris ou en allure, lesquelles existent dans l'instrument de la voix, parmi les langages et quelquefois chez un. À côté d'ombre, opaque, ténèbres se fonce peu : quelle déception, devant la perversité conférant à jour comme à nuit, contradictoirement, des timbres obscurs ici, là clair (Mallarmé, 1992 : 273-274).

5 N'est-ce pas là la leçon baudelairienne des correspondances: «Les parfums, les couleurs et les sons se répondent " (Baudelaire, 1986: 34) ? Il sera donc nécessaire de parler aux étudiants, pour reprendre les termes de Ghyka, de « la texture des mots: timbre et charpente » (Ghyka, 1949 : 39), c'est-à-dire de ce qui rend le mot attrayant ou affectivement plus idoine à l'écoute. Son âme, en quelque sorte, son identité, ou pour reprendre l'idée d'Ivan Fónagy son sexe : si certaines voyelles sont mâles, les vélaires comme /a/ et /o/ et $/ \mathrm{u} /$, les voyelles antérieures sont considérées femelles [e, i, $œ]$ (Fónagy, $1983: 57^{9}$ ). Les mots prennent alors un autre aspect, bien entendu. Il faut aussi revenir aux règles de la versification et plus précisément à Maurice Grammont quand il 
aborde les effets que l'on peut obtenir à travers les sons, là où il parle d'harmonie

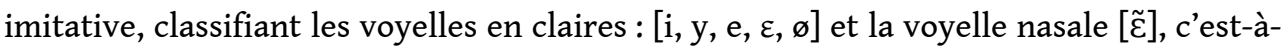
dire les voyelles qui se trouvent en position antérieure du palais. Grammont dit qu'elles sont plus légères que les graves : «Elles sont donc aptes à exprimer un bruit ténu, clair, un murmure doux et léger " (Grammont $1965: 129)$, et « grâce à leur légèreté et à leur douceur, les voyelles claires sont particulièrement désignées pour exprimer des idées légères, gaies, riantes, douces, gracieuses, idylliques » (ibid. : 130). Les voyelles [i] et [y] sont plus particulièrement des voyelles que Grammont définit comme aiguës, qu'il s'agisse "d'un bruit, d'un cri ou d'un sentiment qui pourrait arracher des cris aigus » (ibid. : 128). Puis, Grammont analyse les voyelles éclatantes, appartenant - malgré leur nom - au groupe des voyelles graves [a, $\supset$, ,, a a,$\tilde{]}]$ et enfin, toujours dans le groupe des graves, les voyelles dites sombres : [o, כ̃, u].

Bien sûr, et cela va de soi, toute théorie qui se veut généralisante des règles phonatoires et symboliques en ce qui concerne les sonorités, doit être prise avec prudence. Pierre Léon rappelle que «tout phonème, lorsqu'il est réalisé peut se charger de symbolisme par référence à sa nature articulatoire et acoustique » (Delbouille, 1984 : 43-44 ; Léon, $1971: 3-18$ ), il est aussi vrai et indéniable, selon Gérald Antoine ${ }^{10}$ (cité par Delbouille, 1984 :57), que « rien n'est plus soumis à la subjectivité et surtout à la contingence que la mélodie poétique ».

7 Dans cette phase de tâtonnements sonores, bien entendu, c'est l'enseignant qui montrera aux apprenants à travers sa propre prononciation toute la teneur des théories de Maurice Grammont.

\section{Mot-ivations}

Reconnaître les sons à travers la diction du poème peut susciter chez l'apprenant de la curiosité et favoriser la reconnaissance des sonorités, comme le souligne Guimbretière (1994 : 70) : « Une telle activité permet à l'apprenant de faire fonctionner sa mémoire et d'opérer un mouvement rétroactif pour effectuer les repérages demandés ».

9 Pourquoi ne pas profiter du pur plaisir du texte oral, récité ou lu? Aimer le mot, y découvrir les trésors cachés que celui-ci recèle signifie aimer le poème. Le poème appris à l'école primaire - et avant, la comptine à la maternelle - s'est enfoui dans les plis de la mémoire, quasi effacé, considéré presque ennuyeux, que l'on ne comprend pas, lointain, absurde dans un monde où tout va si vite. Le temps de la lecture avec ses pauses, ses accents, ses rythmes, peut transformer le tout. Tout mot poétique peut renouveler sa sémantique grâce à l'évocation incantatoire; plus le poème sera aimé, plus il y aura une application à bien le prononcer. La mimésis y jouera bientôt un rôle important mais, au début, la lecture devra se faire sans aucune constriction: «Si on demande à l'apprenant d'écouter, on ne doit pas en même temps lui demander de produire» (ibid.: 76).

10 Les savoirs qui développeront les savoir-faire communicatifs (ibid. : 71) pourront enfin s'épanouir : sur le plan phonologique, d'abord, au niveau prosodique ensuite, et plus tard, sur les plans morphosyntaxique, lexical et culturel, quand l'oralité se déplacera vers l'écrit.

11 Ce que nous appelons phonétique n'est en réalité qu'une mélodie de signifiants, et le poème pourrait être son prophète. Le poète chante les phonèmes de son cœur, qu'il 
transforme en images harmonieuses et rythmées. C'est ce qui, peut-être, va permettre également une approche différente et moins pernicieuse de l'étude des sons. Le fait de mal parler une langue provoque de toute évidence un malaise chez l'apprenant qui a quelques difficultés à s'exprimer devant ses collègues, sinon en conservant les défauts d'une mauvaise prononciation apprise lors des cours de français précédents. De la même façon que le poème atteint le cœur et les secrets des adolescents, nous proposerons une lecture individuelle suivie d'une lecture collective, d'abord silencieuse, puis à haute voix: de libre création sonore, la difficulté phonétique, individuellement, deviendra progressivement prononciation correcte: si les sons les plus laborieux, les phonèmes les plus ardus sont mis en musique poétique par ceux qui ont de grosses difficultés, bien prononcer devient un défi envers soi-même et non plus une adaptation imposée par la norme scolaire. Nous paraphraserons le poème d'Eugène Guillevic (2004 : 80), quand il écrivait que : « Être poète / C'est garder ses dix ans / Tout en faisant sienne / La langue des autres ». Nous ne serons pas poète, du moins, certains ne le seront pas, mais tous, acquerront la langue des autres.

C'est ce qu'Anne Godard appelle une pédagogie de la créativité: «explorer les potentialités de la langue, développer des aptitudes à la lecture de fiction, encourager une relation affective à la langue peuvent être des objectifs en FLE aussi bien qu'en FLM d'autant plus que créativité et efficacité ne s'opposent pas » (Aubert-Godard, $2015: 61$ ).

\section{Exercices poétiques}

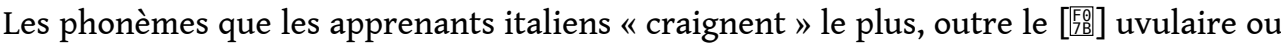
grasseyé ${ }^{11}$, sont, sans aucun doute, les voyelles nasales. Aussi commencerons-nous notre cours par un petit poème de Mallarmé. Notre but initial est de saisir la beauté d'un vers, non pas de le comprendre ou de savoir qui en est l'auteur. Tout cela arrivera peu après, mais d'abord nous nous concentrerons sur les sonorités et la richesse sonore du mot-poème, en nous contentant avant tout de sensibiliser les apprenants au plaisir du texte ou plutôt au plaisir du dire Sainte :
À la fenêtre recelant / Le santal vieux qui se dédore / De sa viole étincelant / Jadis avec flûte ou mandore,//Est la Sainte pâle, étalant/Le livre vieux qui se déplie / Du Magnificat ruisselant/Jadis selon vêpre et complie :// À ce vitrage d'ostensoir / Qui frôle une harpe par l'Ange / Formée avec son vol du soir / Pour la délicate phalange // Du doigt que, sans le vieux santal/Ni le vieux livre, elle balance/Sur le plumage instrumental,/ Musicienne du silence. (Mallarmé, $1945: 53-54)$

On peut aussi considérer le problème des [y], l'opposition entre [y] et [u] :

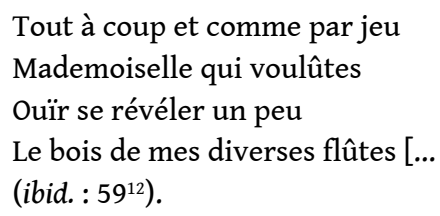

mais toujours dans un souci de pure mélodie, et après Mallarmé, ces quelques vers de Paul Valéry (1958 : 3-4) :

Assise, la fileuse au bleu de la croisée

Où le jardin mélodieux se dodeline ;

Le rouet ancien qui ronfle l'a grisée. 
Lasse, ayant bu l'azur, de filer la câline chevelure, à ses doigts si faibles évasive, Elle songe, et sa tête petite s'incline. Un arbuste et l'air pur font une source vive Qui, suspendue au jour, délicieuse arrose De ses perles de fleurs le jardin de l'oisive. Une tige, où le vent vagabond se repose, Courbe le salut vain de sa grâce étoilée, Dédiant magnifique, au vieux rouet, sa rose. Mais la dormeuse file une laine isolée ; mystérieusement l'ombre frêle se tresse Au fil de ses doigts longs et qui dorment, filée ${ }^{13}[. .$.$] .$

Si, ici, ce choix de vers-phonèmes, semble mettre de côté le poème dans son intégralité, la lecture initiale, faite par l'enseignant sera réalisée en lisant tout le poème. Puis, nous prendrons nos ciseaux mentaux et nous découperons pour le plaisir des sons et pour ne pas inquiéter trop les apprenants. Certes, Valéry et Mallarmé peuvent sembler ardus pour des apprenants mais le but est tout simplement musical, la mélodie des vers doit retenir l'attention, frapper l'imagination, et les vers doivent se déployer dans toute leur beauté harmonieus $\mathrm{e}^{14}$. Il ne s'agit pas, dans un premier temps, de comprendre le poème, il s'agit d'assimiler les sonorités, comme une mélodie murmurée ou un peu comme faisait au théâtre Dario Fo avec son grammelot, mais dans notre cas les sons sont, bien entendu, plus articulés. Nous pouvons aussi tenter de traduire le poème entièrement et le donner à lire dans sa traduction pour mieux accrocher l'attention et pour restreindre les difficultés de lecture pour les apprenants italiens, mais ceci reste une option; notre idée est tout d'abord ancrée dans les sons français. Nous trouvons donc des nasales et des oppositions phonologiques $[e / \varepsilon]$, nous pouvons jouer sur jardin/jardine, câlin/câline, rouet/grisée, ancien/songe, mystérieusement/l'ombre. L'apprenant aura du mal à lire et probablement à comprendre le sens, mais ce qui importe, nous le répétons, c'est qu'il se laisse transporter par la musicalité de l'ensemble. Les vers sur la page (ou sur le tableau) sont lus individuellement puis collectivement. Nous pouvons également chantonner le rythme de l'alexandrin, sans trop rentrer dans les détails métriques - $d u$ moins dans une première phase simplement pour montrer, pour dessiner oralement sans les mots, la mélodie.

Nous pouvons lire un autre poème de Valéry, «L'insinuant », en nous concentrant plus particulièrement sur le dernier quatrain: "Ô Courbes, Méandre,/Secrets du menteur, / Je veux faire attendre / le mot le plus tendre » (Valéry, 1958: 84 $4^{15}$ ).

Mais nous pouvons aussi leur faire entendre d'autres mots tendres, à savoir ceux de la chanson. À ce propos, l'enseignant peut entremêler à la lecture des poèmes, des chansons pour montrer les divergences entre le vers alexandrin et la chanson, souvent octosyllabes ou heptasyllabes: un exemple pour le "lalala" initial pourrait être $L a$ chansonnette de Jean Dréjac (2006 : 83) et même s'il s'agit ici d'alexandrins, le découpage du chant fait ressentir autre chose :

La, la, la, mine de rien/La voilà qui revient/La chansonnette/Elle avait disparu / Le pavé de ma rue / Était tout bête / Les refrains de Paris / Avaient pris l'maquis / Les forains, l'orphéon / La chanson d'Macky/Mais on n'oublie jamais/Le flonflon qui vous met/Le cœur en fête/Quand le vieux musicien / Dans le quartier / Vient revoir les anciens / Faire leur métier / Le public se souvient / D'la chansonnette / tiens, tiens... 
19 Avant d'affronter l'histoire littéraire en parlant de Mallarmé et de Valéry ${ }^{16}$, pourquoi ne pas commencer par raconter l'époque où cette chanson passait sur les ondes, où la plupart des apprenants probablement n'étaient pas encore nés. La narration d'un passé chanté peut intéresser plus que le silence de Valéry, mais plus tard on trouvera les anecdotes justes et « accrocheuses » pour retenir l'attention. Enfin, la chanson est plus près des cœurs et du langage des apprenants, elle est immédiate, et cela peut aider à détendre l'atmosphère (la «honte» des difficultés, le malaise de devoir se perfectionner, etc.).

Voyons maintenant un poème de Ghérasim Luca (1985:61-62), du moins un extrait d'un long poème qui s'appelle « La morphologie de la métamorphose » :

C'est avec une flûte / c'est avec le flux fluet de la flûte / que le fou oui c'est avec un fouet mou / que le fou foule et affole la mort de // La mort de la mort de / c'est l'eau c'est l'or c'est l'orge / c'est l'orgie des / c'est l'orgie des os dans la fosse molle / où les morts flous flottent dessus / comme des flots // Le fou est ce faux phosphore qui coule / phosphore qui cloue la peau du feu / aux eaux aux flots de la porte / alors que la mort de la mort / de la mort morte et folle / n'est que le lot le logis de la faute / qui fausse la logique de loup doux / de la forme / de la forme en forme de mot en forme de mort/en forme de phosphore mort / qui flotte audessus de la fausse forme / c'est le loup du faux cette forme / le faux loup qui fait qui ferme / les fausses portes / qui coule sous la fausse faute / et qui fout qui fout qui fouette / la peau d'eau de la mort // La mort la mort morte en faux / en forme de flot qui flotte / au cou de la forme / eau forte et phosphore doux / âme molle de l'effort de l'or / de l'or mou de l'amorphe // La logique de l'amorphe / fouette et foule l'analogie folle / elle la fouette dans sa fausse loge / qui est en or comme / en or comme l'horloge qui orne/le logis d'un mort// Mais le mort le mot d'or d'ordre / le mot le mot d'or d'ordre / de la mort de la mort / c'est mordre c'est mordre les bornes de la forme / et fondre son beau four dans le corps de la femme [...].

21 Ici l'apprenant peut s'en donner à cœur joie avec les signifiants de ce poème, et corriger la prononciation quand elle est erronée en suivant les indications de l'enseignant. La phonologie des oppositions est présente, et les résultats peuvent être absolument positifs pour acquérir et/ou renforcer de nouvelles habitudes phonatoires.

Pénultième promenade poétique avec Jean Tardieu (2003 : 944), immense jongleur de mots, avec ce petit poème en prose intitulé « Les mots de tous les jours » :

Il faut se méfier des mots. Ils sont toujours trop beaux, trop rutilants et leur rythme vous entraîne, prêts à vous faire prendre un murmure pour une pensée,

Il faut tirer sur le mors sans cesse, de peur que ces trop bouillants coursiers ne s'emballent.

J'ai longtemps cherché les mots les plus simples, les plus usés, même les plus plats. Mais ce n'est pas encore cela : c'est leur juste assemblage qui compte.

Quiconque saurait le secret usage des mots de tous les jours aurait un pouvoir illimité - et il ferait peur.

Ce poème est un très bon exercice de lecture et un puits de sémantique poétique. Tardieu, bien sûr, est une source inépuisable, car il peut être très utile aussi en ce qui concerne la décomposition du langage, les fautes grammaticales, les défauts physiques de prononciation et autres phénomènes langagiers.

Dans le cadre de la lecture des sonorités, là où le poème semble n'être qu'un emprunt momentané, les sons les plus « défectueux » des apprenants peuvent être corrigés, et les apprenants pourront lire un autre poème, celui-ci de Léon-Paul Fargue (1967 : 155-156), qui sous le titre léger de «Chanson » déploie un éventail de sonorités : 
Les fabricants ont arrangé / Pour notre usage, les objets/Usuels - les objets aimés... // Le bruit du cristal éveillé / Pareil à un sommeil léger / N’a pas troublé n'a pas troublé/Les gens-de leur prospérité...//Ils en ont fait des quantités / Sans être émus de leur beauté / Et pour satisfaire à la vente, / Notre petite sœur la lampe, / La lampe qui voit nos baisers... // Notre petite sœur la lampe / À la ronde voit nos baisers. / Comme les morts elle dormait / Sans bruit, au creux d'un tertre vert... // Tout le jour elle était fermée/Sur son rôle et se recueillait / Et se taisait comme se tait/Une ruche, sans bruit l'hiver... // Mais voici l'heure. Une petite/Étoile tremble et périclite... Au bleu triste de la croisée / La mouche tait son bruit disert... // Et la lampe fait sa lumière / Douce et pâle, couleur des plages, / Couleur des blés, couleur des sables, / Couleur des sables du désert... // Dans une maison qu'on ignore / le soir monte au bras du danger / Et s'arrête sur un palier / Devant une porte marquée.

\section{Joindre le ludique au poétique : les jeux du bazar, le je et le hasard}

« Il y a des vers qu'on trouve. Les autres, on les

fait. "

Paul Valéry, Tel quel

Avec cette dernière partie, nous proposons un jeu poétique et créatif : à partir d'un premier vers (transcrit phonétiquement ici), en se laissant guider par les sonorités et 
leurs propres goûts, apprenants et enseignants peuvent s'amuser à composer. Nous suggérons quelques traces :

Les embruns de la mer éclaboussent les oursins

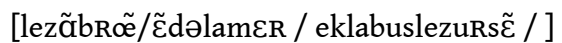

Les emprunts du maire débroussent les prêts des marins

Les gens bruns de la mairie blousent les saouls et les saints

Les ennuis de la mère prennent le bus du matin

Les ancêtres de l'amer drainent le jus du matin

Les poudrins de l'amer hument les jours incertains

L'amertume du café hier floue en vain les malins

Lait ambre et teint de la mère éclats doux du lin

Léandre au teint ambré rêve aux raouts de juin

Les flaques de la flotte de la mer fument le purin

Lézard brun du mur rêve sur la rousse douce fleur du matin

Etc.

29 Et l'on pourrait continuer à l'infini. Ici, c'est la poésie qui doit prendre le pas sur les sciences des sons, au grand dam de la phonétique et/ou de la phonologie. Peu importe les sons à travailler, il s'agit de laisser une plus grande liberté aux apprenants dans leurs choix des mots, qu'ils apprennent à construire le poème, les rythmes, les images et, bien sûr, les sons, mais du fond de leur esprit et/ou de leur cœur. On pourrait objecter que dans une classe d'apprenants il n'est pas question de trouver des poètes. Qu'à cela ne tienne, c'est le jeu poétique, la création, qui compte. On peut devenir apprenti-poète sans le savoir et, en attendant, on partage une langue étrangère, tout d'abord oralement. Puis on se dirige progressivement vers la composante scripturale ${ }^{17}$ : «Toutefois, le travail systématique de prononciation sera d'une efficacité limitée s'il n'y a pas ouverture vers l'oral spontané et vers l'écrit ", écrivait dans l'introduction à sa méthodologie Monique Callamand (1981:9), et c'est, nous semble-t-il, ce que nous avons cherché à faire.

\section{Conclusion}

N'étant ni phonéticien, ni didacticien, l'approche que nous avons voulu effectuer est purement liée à notre propre enseignement et, bien entendu, à l'amour pour la poésie, trop souvent marginalisée. Il est vrai que l'on reconnaît à celle-ci, de plus en plus, un rôle important en classe de FLE. Élisabeth Guimbretière (1994 : 83) en parle : « Le poème parce qu'il donne le pouvoir de jouer avec les sonorités est une ressource pédagogique inestimable. C'est le lieu ou l'enseignant doit totalement s'effacer pour laisser se faire l'évocation et la représentation mentale des sons : le pouvoir évocateur de la poésie va servir la mémorisation des sonorités ${ }^{18}$ ". C'est bien la sorcellerie évocatoire dont parlait Baudelaire, ou presque. Bachelard (1989:43) - il faut bien le citer - disait qu'il faut écouter le son des mots comme un enfant qui écoute la mer dans son coquillage collé à l'oreille. La fantaisie des mots virevoltant avec leurs sonorités peut favoriser la création individuelle et l'envie d'apprendre.

La poésie est donc présente dans la didactique des langues même si, comme le remarquent Auréliane Baptiste, Donatienne Woerly et Olivier Lumbroso (2015:179), " presque tous compris entre 1915 et 1970, ces poèmes pris comme hypotextes sont, dans les ouvrages de FLE, ramenés à Prévert et Queneau quasi exclusivement ». Ceci s'explique facilement par le fait indéniable que l'approche poétique pour des apprenants qui lisent peu, et encore moins la poésie, se réalise plus facilement quand à 
la rime on ajoute le jeu. Jeux de rimes rime avec apprendre et sourire (nous allions écrire sourime) mais l'on pourrait tout aussi bien prendre des poèmes d'amour puisque l'amour après le jeu sont les thèmes les plus récurrents de notre post-modernité. Il est vrai que le choix des poètes est immense et, une fois partis dans ce long voyage, l'on peut aisément (re)découvrir d'autres poèmes moins ludiques, certes, mais où les signifiants sont porteurs de sens car le signifiant nous ramène immédiatement à la bonne prononciation, c'est ce que nous avons tenté de montrer dans le cadre de cet article. Phonématique, phonologie, phonétique et prosodie, la langue est bien « une combinaison des sons" (Chiss, Filliolet et Maingueneau, 2012:77); cette combinaison est celle qui fera naître l'émotion chez l'apprenant une fois qu'il se rendra compte du parcours effectué. Dans son étude sur l'oralité-auralité, Ève-Marie Rollinat-Levasseur (2015 : 237) a écrit :

Lorsque l'apprenant lit à haute voix, il s'entend et s'autoévalue, de la même façon que le fait un chanteur : l'oralité, ici le fait de lire oralement, est ainsi le premier stade de prise de conscience de la production orle dans la langue cible, ce qui permet, au moment du déchiffrage de la suite du texte et de sa relecture, de s'appuyer sur cette perception pour se corriger et améliorer sa performance orale. La poésie nous parle, il suffit de bien vouloir l'écouter. À son tour, la poésie a le don d'être écoutée et lue, et entre l'écoute et la lecture, elle sait aussi écouter les voix, toutes les voix, la nôtre et celles des autres. Elle est vraiment le juste médiateur qui ouvre les portes de la communication.

\section{BIBLIOGRAPHIE}

Bachelard G., 1989 [1960], La poétique de la rêverie, Paris, PUF.

Baptiste A., Woerly D. et Lumbroso O., 2015, « Chapitre 4 : Le rôle de la littérature dans les apprentissages langagiers : de l'écriture créative à la conscience de la langue », in A.Godard (dir.), La littérature dans l'enseignement du FLE, Paris, Didier, coll. « Langues et didactique », p. 169-219.

Baudelaire C., 1986, Les Fleurs du mal, Paris, José Corti.

Bécaud G., 1995, Alors raconte... L'intégrale de ses chansons, Paris, Librairie Générale Française.

Callamand M., 1981, Méthodologie de l'enseignement de la prononciation : organisation de la matière phonique du français et correction phonétique, Paris, CLE International.

Castellotti V., 1997, « Langue étrangère et français en milieu scolaire : didactiser l'alternance », ELA (Études de linguistique appliquée), no 108, p. 401-410.

Chiss J.-L., Filliolet J. et Maingueneau D., 2012 [1993], Introduction à la linguistique française, Paris, Hachette, tome 1.

Delbouille P., 1984, Poésie et sonorités II. Les nouvelles recherches, Paris, Les Belles Lettres.

Dréjac J., 2006, Comme elle est longue à mourir ma jeunesse, Paris, Christian Pirot.

Duverger J., 2005, L'enseignement en classe bilingue, Paris, Hachette FLE. 
-, 2007, « Didactiser l'alternance des langues en cours de DNL », Tréma [En ligne], no 28, p. 81-88. Disponible sur : <https://journals.openedition.org/trema/302>.

Fargue L.-P., 1967 [1943], Poèmes suivi de Pour la musique, Paris, Gallimard.

Fónagy I., 1983, La vive voix. Essais de psycho-phonétique, Paris, Payot.

Genette G., 1969, Figures II, Paris, Éditions du Seuil, coll. « Tel Quel ».

Gérald A., 1982, Vis-à-vis ou le double regard critique, Paris, PUF, coll. «Écriture ».

Ghyka M. C., 1949, Sortilèges du verbe (préface de Léon-Paul Fargue), Paris, Gallimard.

Godard A., 2015, « Chapitre 2 : Enjeux de la formation littéraire aujourd'hui », in A.Godard (dir.), La littérature dans l'enseignement du FLE, Paris, Didier, coll. « Langues et didactique », p. 56-90.

Grammont M., 1965 [1937], Petit traité de versification française, Paris, Armand Colin.

Guillevic E., 2004, Présent : poèmes 1987-1997, Paris, Gallimard.

Guimbretière É., 1994, Phonétique et enseignement de l'oral, Paris, Didier Hatier.

Jakobson R., 1963, Essais de linguistique générale, trad. par Nicolas Ruwet, Paris, Éditions de Minuit, tome 1.

Lauret B., 2007, Enseigner la prononciation du français : questions et outils, Paris, Hachette, coll. «f $»$.

Léon P., 1971, «Éléments phonostylistiques du texte littéraire », in P. Léon, H. Mitterand et R. Nesselroth (dir.), Problèmes de l'analyse textuelle/Problems of Textual Analysis, MontréalParis-Bruxelles, Didier, p. 3-18.

Luca G., 1985, Héros-limite, Paris, José Corti.

Mallarmé S., 1945, Euvres complètes (édition de G. Jean-Aubry et H. Mondor), Paris, Gallimard, coll. « Bibliothèque de la Pléiade ».

-, 1992, CEuvres (édition d'Yves-Alain Favre), Paris, Classiques Garnier-Bordas.

Pope A., 1881, Essai sur la critique, Paris, Hachette. Disponible sur : <https://gallica.bnf.fr/ark:/ 12148/bpt6k5813018t.texteImage>.

Proust M., 1954, Du côté de chez Swann, Paris, Gallimard.

Rollinat-Levasseur È.-M., 2015, « La littérature en acte : voir, entendre, ressentir », in A.Godard (dir.), La littérature dans l'enseignement du FLE, Paris, Didier, coll. « Langues et didactique », p. 220-264.

Sartre J.-P., 1948, Situations II, Paris, Gallimard.

Tardieu J., 2003, CEuvres (édition de J.-Y. Debreuille), Paris, Gallimard, coll. « Quarto » (Pages d'écriture, 1967).

Valéry P., 1958, Poésies, Paris, Gallimard, coll. « Poésie ».

-, 2001, « Rhumbs » (1943), dans Tel quel, Paris, Gallimard, coll. « Folio essais ».

\section{NOTES}

1. Il s'agit donc de jeunes étudiants italiens, en général, âgés de dix-huit/vingt ans (mais l'âge peut varier avec des étudiants plus âgés) qui, au collège et au lycée, ont fait quelques années de 
français, dans une moyenne que l'on peut situer entre 3 et 8 ans. Avec un bon pourcentage (de $10 \%$ à $15 \%$ mais, ces dernières années, nous allons résolument vers $20 \%$ ) d'étudiants qui n'ont jamais fait de classe de français. Le niveau, selon le Cadre européen commun de référence pour les langues (CECRL) correspond, grosso modo, à un A2 et après un cursus de 3 ans (entre les heures de laboratoires et les cours), le niveau n'atteint pas le B1 (sauf quelques exceptions qui ont un B2). Lire et apprécier Mallarmé deviendraient alors pour enseignant et apprenants un vrai défi.

2. «À savoir qu'une voyelle dite aiguë, comme le / y / semi-consonne et le / i / de nuit, peut évoquer, par une synesthésie naturelle, une couleur claire ou une impression lumineuse, et qu'au contraire une voyelle dite grave, comme le / u / de jour, peut évoquer une couleur sombre, une impression d'obscurité » (Genette, 1969 :101-122).

3. Nous disons "classe» dans le sens le plus ample du terme, il s'agit bien de cours et de laboratoires universitaires.

4. Matila C. Ghyka, Sortilèges du verbe (préface de Léon-Paul Fargue), Paris, Gallimard, 1949.

5. «Le nom de Parme, une des villes où je désirais le plus aller depuis que j'avais lu la Chartreuse, m'apparaissait compact, lisse, mauve et doux. [...] puisque je l'imaginais seulement à l'aide de cette syllabe lourde du nom de Parme, où ne circule aucun air, et de tout ce que je lui avais fait absorber de douceur stendhalienne et du reflet des violettes. Et quand je pensais à Florence, c'était comme à une ville miraculeusement embaumée et semblable à une corolle, parce qu'elle s'appelait la cité des lys et sa cathédrale, Sainte-Marie-des-Fleurs » (Proust, 1954 : 463).

6. «Florence est ville et fleur et fleur et femme, elle est ville-fleur et ville-femme et fille-fleur tout à la fois. Et l'étrange objet qui paraît ainsi possède la liquidité du fleuve, la douce ardeur fauve de l'or et, pour finir, s'abandonne avec décence et prolonge indéfiniment par l'affaiblissement continu de l'e muet son épanouissement plein de réserves. À cela s'ajoute l'effort insidieux de la biographie. Pour moi Florence est aussi une certaine femme, une actrice américaine qui jouait dans les films muets de mon enfance et dont j'ai tout oublié, sauf qu'elle était longue comme un long gant de bal et toujours un peu lasse et toujours un peu chaste, et toujours mariée et incomprise, et que je l'aimais, et qu'elle s'appelait Florence.» (Sartre, 1948 : 66-67).

7. «C'est trop peu que l'oreille ne soit offensée d'aucune dureté, / le son doit paraitre un écho de la pensée./Douce est l'harmonie quand souffle le zéphyr caressant; faites couler une onde paisible en vers plus coulants encore. »; "Ce n'est pas assez que / nulle dureté / ne donne blessure / le son doit/paraître un écho à (de) la pensée./L'accent est doux/lorsque le zéphyr / souffle doucement / et le ruisseau uni / coule dans des vers / plus-unis encore » (Pope, 1881 : 42-43 ; Jakobson, 1963 : 240).

8. Dans notre lecture poétique et sa compréhension textuelle, nous montrerons comment l'on peut se situer entre les deux courants pour une bonne traduction.

9. À travers les métaphores «on leur attribue des qualités émotives et morales: certaines voyelles sont vulgaires, d'autres distinguées ou "mignardes"; telle consonne est ressentie comme efféminée, telle autre comme virile » (Ivan Fónagy, 1983 : 57, cité par Guimbretière, 1994 : 56 ).

10. Cf. Antoine Gérald, 1982, Vis-à-vis ou le double regard critique, Paris, PUF.

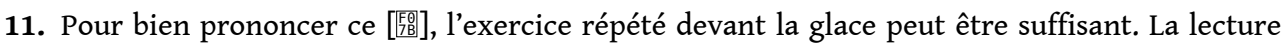
ensuite (avec ses oppositions) aidera, voire renforcera; le poème ci-dessus de Mallarmé recèle (c'est le cas de le dire) indubitablement ce son. Il reste aussi la possibilité de faire écouter, ensuite, une chanson d'Édith Piaf avec ses / r / apicaux ou roulés, que d'ailleurs, les apprenants italiens connaissent bien puisque leur prononciation de cette consonne est roulée. D'autre part, cette écoute peut aussi permettre une approche sociolinguistique de la langue et donc tout ce qui a trait à la variation diatopique, diachronique, diastratique et diaphasique.

12. La suite de Feuillet d'album : «Il me semble que cet essai / Tenté devant un paysage / À du bon quand je le cessai / Pour vous regarder au visage // Oui ce vain souffle que j'exclus / Jusqu'à la 
dernière limite / Selon mes quelques doigts perclus / Manque de moyens s'il imite // Votre très naturel et clair / Rire d'enfant qui charme l'air. »

13. La fin du poème «La fileuse »: "Le songe se dévide avec une paresse / Angélique, et sans cesse, au doux fuseau crédule, / La chevelure ondule au gré de la caresse... // Derrière tant de fleurs, l'azur se dissimule, / Fileuse de feuillage et de lumière ceinte : / Tout le ciel vert se meurt. Le dernier arbre brûle. // Ta sœur, la grande rose où sourit une sainte, / Parfume ton front vague au vent de son haleine / Innocente, et tu crois languir... Tu es éteinte / Au bleu de la croisée où tu filais la laine."

14. On peut glisser entre ces deux poètes quelques vers de Verlaine; nous pensons, bien entendu, à « Il pleut sur la ville».

15. Nous transcrirons aussi les trois premiers quatrains : «Ô Courbes, méandre, / Secrets du menteur, / Est-il art plus tendre/Que cette lenteur?//Je sais où je vais,/Je t'y veux conduire, / Mon dessein mauvais/N'est pas de te nuire...// (Quoique souriante/En pleine fierté, / Tant de liberté / La désoriente !) // [...]».

16. Le choix de poètes se fait à partir des mots et non pas des idées, selon ce que Mallarmé disait à Degas. L'idée qu'il s'agit de poètes difficiles est bien ancrée et, d'une certaine façon, n'est pas fausse, mais, seulement, si l'on essaie d'expliquer le sens du poème, car le mot seul est avant tout signifiant. Cela nous permet aussi de sortir des «sentiers (re)battus » de Prévert et de Queneau, même s'ils peuvent paraître (et en fait, ils le sont) plus attachants aux yeux de nos lecteurs modernes, mais il nous semble, pour Mallarmé et Valéry, qu'il s'agisse d'une autre « sorcellerie évocatoire » à laquelle nous ajouterons l'épithète « incantatoire ».

17. Pour l'écrit, vu que nous sommes toujours dans l'esprit de la création poétique, nous pouvons démarrer sur les chapeaux de roue avec une chanson qui décompose et recompose les signifiés à partir des signifiants. Il s'agit d'une chanson écrite par Louis Amade et Pierre Delanoë sur une musique de Gilbert Bécaud et dont le titre est : «Croquemitoufle » (1958). Pour donner une idée, voici la première strophe « écrite » : «Je me rencroquemitoufle / Au fond des pantoufles / Quand tu n'es pas là / Et je m'éfiléliloche / Comme un fond de poche / Quand tu n'es pas là / Je périclite, décline/En carabobine/Qui n'en finit pas/Et la vie me semble fouine/Blette et filandrine / Quand tu n'es pas là, // oh la la / Que le temps s'étire / Oh la la / Tirelirela [...] » (Bécaud, 1995 : 86-87). Pour procéder à la vérification de la compréhension de la chanson - mais aussi à sa présentation avec ou sans écoute -, il vaudrait mieux se munir d'un bon dictionnaire.

18. C'est Guimbretière qui souligne.

\section{RÉSUMÉS}

Constatant que nos étudiants italiens de FLE ont contracté, au cours des années, de mauvaises habitudes de prononciation, souvent dues à un enseignement lacunaire de la connaissance correcte des sons, et que le temps d'enseignement universitaire (les laboratoires linguistiques) est réduit d'année en année (les langues semblent perdre leur charme au profit de sciences « inexactes » en ce qui concerne les futures promesses de débouchés), notre propos est de chercher à récupérer le temps perdu en insistant, au début de l'année d'un cours de Langue et Traduction française, sur l'oralité. Partant du poème et du jeu sur les signifiants et jonglant avec les signifiés, nous essayerons de motiver les étudiants, peu enclins à la lecture de la poésie et qui, en outre, négligent de se mettre quotidiennement devant un miroir durant quatre ou sept minutes, temps prescrit par les méthodes phonétiques et phonologiques, et nécessaire pour 
acquérir de nouvelles habitudes phonatoires. Une partie de cet article sera dédiée aux poètes (Mallarmé, Valéry, Tardieu, Prévert, Luca) pour montrer comment la force orale du poème peut devenir un atout majeur pour une bonne prononciation ; une autre, à partir d'un vers, sera une sorte de « cahier de création » phonique, sémantique voire poétique.

Our proposal originates from the observation that our Italian FLE students have assimilated a wrong French pronunciation over the years. If, on the one hand, language training often fails to provide the students with an adequate knowledge of sounds and phonemes of French language, on the other hand, at university the amount of time dedicated to languages (languages' workshops) is getting ever shorter, as languages seem to lose their appeal to the benefit of 'exact' sciences which eventually prove 'inexact' as far as job opportunities are concerned. In light of the above, our proposal aims at making up for lost time, and to work particularly on orality at the beginning of the year with a course on « Langue et Traduction française ». Starting from poetry and playing with signifiers (signifiants) and meanings (signifiés), we will endeavour to motivate the students to do what they are usually less inclined to do : reading poetry and pronouncing French in front of a mirror for 4 to 7 minutes a day - the prescribed time by phonetics' methods in order to acquire new phonetic habits. A part of this article will be dedicated to the selection of poets (Mallarmé, Valéry, Tardieu, Prévert, Luca) in order to show how the oral force of a poem can become a valid asset for a good pronunciation. In another part, the activities will set off from a verse, which will act as a sort of a phonic and semantic - that is, poetic - « cahier de création ».

\section{INDEX}

Mots-clés : poème, signifiants, signifiés, oralité, habitudes phonatoires.

Keywords : poetry, signifiers, meanings, orality, phonetic habits.

\section{AUTEUR \\ RENÉ CORONA}

Università di Messina 